\title{
Research of Magnesium-based Alloys for Medical Applications
}

\author{
Cheng Jingtao
}

The Engineering and Technical college of Chengdu University of Technology, Leshan 614007, China

Keywords: magnesium alloys, biomedical, research

\begin{abstract}
The biodegradable magnesium and its alloys are a focus of degradable biomaterials research. Their modulus and density approach the human bone's. As implant materials, they can reduce the shielding of implants. They are also lighter than other medical metal. So, they are suitable materials for biodegradable orthopedic implants and vascular stents. But they are difficult to process, corrode rapidly, need a better biocompatibility. This paper reviews the current research status on magnesium and its alloys as biomedical materials. Their main problems and improvements as biomedical materials are introduced. Their directions as biomaterials are also briefly described.

Author introduction.
\end{abstract}

\section{Introduction}

With the development and progress of economic and social society, biomedical materials have broad market and good development, in which the implant materials are especially striking. There are mainly three kinds of biological implant materials: metal materials, ceramic materials and polymer materials. Because of the good performance, metal materials have been widely used in many medical fields, especially in the orthopedic field.

Medical and implantable metal materials now commonly used are: stainless steel, titanium alloy and cobalt-chromium alloy, etc., and they have great advantages as the implanted material, but the biggest drawback is non-biodegradable, so they needed to be taken out by reoperation after completing its mission. Therefore, the research on biodegradable implant metal materials was born at the right moment. In the 1930s, magnesium alloy has been found biodegradable in the human body. Therefore, magnesium alloys become the study hotspot in the field of medical implant materials. Compared with biodegradable polymer material, magnesium alloys have good mechanical compatibility, and can provide higher initial stability and initial support. The specific strength of pure magnesium is $133 \mathrm{GPa} /(\mathrm{g} / \mathrm{cm} 3)$, and the specific strength of magnesium alloy with super strength is $480 \mathrm{GPa} /(\mathrm{g} / \mathrm{cm} 3)$, higher than that of Ti6Al4V $(260 \mathrm{GPa} /(\mathrm{g} / \mathrm{cm} 3))$ nearly 1 times. Compared with traditional biomedical metal (stainless steel, cobalt chromium alloy, titanium alloy, etc.), modulus of elasticity (45GPa) and density $(1.74 \mathrm{~g} / \mathrm{cm} 3)$ is closer to human body skeleton (20GPa, $1.75 \mathrm{~g} / \mathrm{cm} 3$ ), after implanted in the body, which can effectively reduce the effect of the stress shielding, and it is lighter relative to other medical metal, suitable for hard tissue implant and tissue engineering scaffold material. Magnesium alloy has good biocompatibility. Magnesium is one of the mineral elements of the human body, and there is nearly $1 \mathrm{~mol}$ magnesium in the adult body, then it almost takes part in all the metabolic processes involved in the human body, close to nerve, muscle and heart function, and it is second only to calcium, potassium and sodium body constant elements.

Because chemical properties of magnesium are lively, and the standard electrode potential is low, in complex ions environment of the human body, oxide film on the surface of the magnesium can be destroyed, leading to the degradation in the body too quick. Magnesium is the close-packed hexagonal crystal structure, the plasticity is relatively poor, increasing processing difficulty of it as the intravascular stent tubing and other aspects. Improving corrosion resistance of medical magnesium alloy and controlling its degradation speed, improving the cold deformation ability of magnesium, preparing porous magnesium and carrying on the modification, are important aspects of enlarging applications of biomedical magnesium alloy.

This paper briefly comments the properties of current biomedical magnesium alloys is, and introduces the main problems of medical magnesium alloys and modification methods to improve performance and extend its application, and prospects some directions of the development of 
biomedical magnesium alloy.

\section{Degradation and control of medical magnesium alloys}

Pure magnesium board is first applied to the leg bone fracture in 1907, but it only exists in the body for eight days. Because the degradation speed is too fast, and the reaction with interstitial fluid produces a large number of hydrogen and bubbles, gathered in the subcutaneous tissue, leading to the implant failure. When medical stainless steel appeared, the research on magnesium alloy as implanted materials stagnated for quite some time. The development of the modern metallurgy technology and surface modification technology made it possible to corrosion of magnesium and controllable degradation, the people can again scan magnesium and alloy in the angle of biodegradable materials, how to control the speed degradation of magnesium and alloy become one of the focuses of medical magnesium alloy researchers.

\subsection{New type alloy development}

The purity content of medical pure magnesium is the most important factor to determine its corrosion resistant performance. In the pure magnesium there are impurities such as $\mathrm{Fe}, \mathrm{Ni}$ and $\mathrm{Cu}$. So by pure purification of smelting, it can greatly reduce content of harmful impurities of heavy metals in magnesium alloys, which can effectively improve the corrosion resistance of magnesium alloys.

Alloying is a way of improving the corrosion resistance of magnesium alloys. Rare earth elements make magnesium alloy microstructure improved, and strengthen the grain boundaries and phase boundaries, thus slow down the corrosion of magnesium alloys in $\mathrm{NaCl}$ solution. $\mathrm{F}$. Witte $\mathrm{et}^{[1]}$ have study corrosion of four kinds of magnesium alloys in the body, the alloy composition has greater effect on the corrosion resistance of magnesium alloys, the corrosion resistance of LAE442 alloy with rare earth element additions such as $\mathrm{La}$, $\mathrm{Ne}$ is much larger than that of $\mathrm{Al}$, $\mathrm{Zn}$ series AZ31 and AZ91 alloy; Their research also suggested that the corrosion resistance of LAE442 and AZ91D alloy was opposite in vivo and in vitro, and in vivo LAE442 had higher corrosion resistance, in vitro had a opposite, which showed that the effects of the alloy composition on alloy corrosion resistance in vivo and in vitro remained to be further clear. The research of Yang Ke group ${ }^{[2]}$ in metal place showed that the addition of $\mathrm{Mn}$ and $\mathrm{Zn}$ can promote magnesium phosphate layer to generate in simulated body fluid, improving the corrosion resistance of magnesium substrate. Pking University Zheng Yufeng group ${ }^{[3]}$ used the characteristics of Ca element in human body, for it is one of the essential mineral elements and has light weight, to develop the $\mathrm{Mg}$ - Ca duplex alloy, and to adjust the corrosion resistance of the alloy by adjusting the content of Ca element. M. Bobby Kannan ${ }^{[4]}$ and others studied on the degradation behavior of calcium magnesium alloy in vitro SBF, and the results showed that calcium magnesium alloy has higher corrosion resistance.

\subsection{Surface modification}

Because the oxide film of magnesium and magnesium alloy surface is loose and brittle, not enough to form protective film which can prevent the further corrosion, as a result, to make it possible that magnesium and magnesium alloy can replace the existing metal biomaterials, must carry out the surface modification for it. Surface modification technologies of magnesium alloys at present basically have: there are core permeability alloying, vapor deposition coating, laser processing, metal coating, anodic oxidation, chemical conversion coating, etc.

As medical degradation implant material, in addition to consider its corrosion resistance to control the degradation rate, medical magnesium alloy surface must have better biocompatibility. In the expansion of infiltration method of alloy, aluminum and nickel can be directly plated on the surface of magnesium metal. However, aluminum and nickel have a long-term potential toxicity to human body, therefore two methods of surface modification do not apply to medical magnesium alloys. The author thinks that the anodic oxidation and chemical conversion coating method are more simple and effective, the generated film composition is controlled, can take into account both corrosion resistance and biocompatibility.

K.Y. Chiu ${ }^{[5]}$ and other researches have shown that the fluoride coating prepared by conversion coating method can effectively improve the corrosion resistance of magnesium in Hank's solution. Huang Jingjing ${ }^{[6]}$ and so on can use dip-coating method on the surface of magnesium to prepare of 
polylactic acid coating, making corrosion resistance of magnesium significantly improved. Gao Jiacheng ${ }^{[7]}$ will make pure magnesium at room temperature in the initial $\mathrm{pH}$ value of 9.3 of supersaturated $\mathrm{NaHCO}_{3}-\mathrm{MgCO}_{3}$ mixed solution soak for $24 \mathrm{~h}$, and the surface is mainly $\mathrm{MgCO}_{3} 3 \mathrm{H}_{2} \mathrm{O}$ crystal. After heat treatment, $\mathrm{MgCO}_{3} 3 \mathrm{H}_{2} \mathrm{O}$ crystals will transform into a mixture of $\mathrm{MgCO}_{3}$ and $\mathrm{Mg}(\mathrm{OH})_{2}$, and they also use liquid phase chemical deposition technology to prepare hydryoxyapetite coating, and after soaking by SBF it found that the two technologies can improve the corrosion resistance of pure magnesium in biomimetic environment. Gong Pei ${ }^{[8]}$ and others self-assemble monomolecular layer on the surface of pure magnesium, and make the monolayer calcification processing, soak treated pure magnesium specimen in saturated solution of calcium phosphate, and obtain hydroxyapatite coating by bionic and deposition. Xu Yue[9] and others use sol-gel method to prepare a non-toxic, non-polluting $\mathrm{CeO}_{2}$ ceramic coating on AZ91D magnesium alloy surface, the corrosion resistance of the samples after the film forming in $\mathrm{NaCl}$ solution is good, and the passivation ability is strong.

Zeng Rongchang ${ }^{[10]}$, plasma thermal spray $\mathrm{TiO}_{2}$ on magnesium alloy AM60, obtaining cellular ceramic coating combined with matrix well. P. Shi ${ }^{[11]}$, first use micro-arc oxidation to prepare porous magnesium ceramic coating layer on magnesium alloy surface, further prepare a layer of $\mathrm{TiO}_{2}$ by sol-gel method to make micro-arc closed, then coating significantly improved its corrosion resistance in Hank's solution. Y.W. Song et al. ${ }^{[12]}$ use electrochemical method to prepare hydroxyapatite coating on the surface of AZ91D, and results show that in SBF the coating effectively reduces the degradation speed of the magnesium alloy.

\subsection{New processing technology}

Rapid solidification method can reduce the influence of harmful elements of magnesium alloy, thus improve the corrosion resistance of magnesium alloy, the reasons are as follows: 1) rapid solidification can make alloy composition and organization even, and inhibit local corrosion;2) rapid solidification can improve the solid solubility of alloy, and make the harmful impurity dissolved in the matrix, not form harmful precipitated phase, reducing the degree of corrosion, but also can form amorphous oxide film, and improve the corrosion resistance.

Grain refinement is benefit to improve the corrosion resistance of magnesium alloy, for $\mathrm{Mg}-\mathrm{Al}$ alloy more tested in the current medical aspects, grain refinement can make its corrosion mechanism turn point corrosion into a surface corrosion, improving its corrosion resistance. After casting, the surface grain of AZ91D alloy gained refinement, and its corrosion resistance is significantly higher than the original ingots. H.Wang ${ }^{[13]}$ through the hot rolling and channel extrusion methods, has refined the AZ31 magnesium alloy grain, after soaking in Hank solution it found that grain refinement can improve the corrosion resistance of alloy, but also can improve the fatigue strength of the alloy.

\subsection{Heat treatment}

With a proper heat treatment, magnesium alloys not only reduces the chance of stress corrosion, and can refine grains, and adjusting organization can improve its corrosion resistance. Tan Xiaowei [14] adjusts the heat treatment process to generate compact MgO style layer with good combination in the pure magnesium specimen surface, and the layer material can improve the corrosion resistance of pure magnesium in simulated body fluid SBF solution. Research of Chenglong Liu ${ }^{\text {[15] }}$ showed that surface tissue equalization can effectively improve the corrosion resistance of magnesium, the corrosion resistance in SBF of AZ63 magnesium alloy after aging increased nearly $1 / 2$ compared with the untreated alloy.

\section{Medical magnesium alloy capillary material and the porous magnesium}

\subsection{Medical magnesium alloys capillary material}

Absorbable intravascular stent is one of the important applications of medical magnesium alloy, but the preparation of coronary artery implant stent needs capillary base material. Magnesium is the close-packed hexagonal crystal structure, and its cold deformation ability is poor. Northwest nonferrous metal research institute ${ }^{[16]}$ used cold-rolled way to process continually $13.5 \mathrm{~mm}$ hot extrusion tube, then magnesium alloy capillary materials were successfully prepared by controlling technology and the heat treating regime. 


\subsection{Porous magnesium}

There are many kinds of preparation methods of porous magnesium, but in order to guarantee the biocompatibility of porous magnesium, we must carefully choose pore-forming agent and preparation methods. Methods currently used in research of magnesium based medical porous material basically have the following several kinds: powder metallurgy method, using laser drilling method, investment casting method, the seepage flow casting method, etc. Porosity and pore size plays a decisive role on the performance of the porous magnesium, adjust the pore size and porosity of porous magnesium through the choice of particle size and content of pore-forming agent, making porous magnesium possess similar elastic modulus and aperture with the human skeletons, to meet the requirements of it as the implanted material for human body. The compression strength and elastic modulus of porous magnesium decreases with the increase of aperture, the cell wall of porous magnesium with small aperture is larger and thinner than that with big aperture, and the specific surface area of the former is larger than the latter, which makes bearing capacity of the cell walls increase, the yield stress of the overall material has improved.

\section{Outlooks}

Although the magnesium alloy has many advantages, suitable for hard tissue implant and tissue engineering scaffold material, but how to control the degradation rate of magnesium alloys, improving its performance and making it real application in the human body would still need a lot of research work. (1) The development new corrosion resistant magnesium alloy should further systematically research the degradation mechanism of magnesium in the body, as well as the interaction of tissue in the body, fully consider that the magnesium alloy has larger difference in the corrosion mechanism in vivo and in vitro, and further study how to use corrosion resistance in vitro test method to guide the development, machining and heat treatment of new medical magnesium alloys. (2) The surface modification of magnesium alloys should, on the basis of keeping the biocompatibility of medical magnesium alloy, research how to improve its biological activity and realize the bio-functionalization of the surface. (3) Research a new preparation methods of porous magnesium, and how to take into account appropriate tissue growth structure with excellent mechanics performance, strengthen the surface treatment of porous magnesium, further study on the interactive relationship with bone tissue. Magnesium alloy applications in the field of biological quote are far from mature, with the wide depth of science and technology progress and research, it must has sustainable development as biological implant materials.

\section{References}

[1] F. Witte, V. Kaese, H. Haferkamp, et al. In vivo corrosion of four magnesium alloys and the associated bone response [J].Biomaterials, 2005, 26: 3557-63

[2] Yan Tingting, Tan Lili, Xiong Dangsheng, etc. Research Progress in Magnesium-based Metals for Medical Applications[J]. Materials Review,2008,22 (1): 110-2,129

[3] Zijian Li, Xunan Gu, Siquan Lou, et al. The development of binary MgeCa alloys for use as biodegradable materials within bone[J]. Biomaterials, 2008, 29: 1329-44

[4] M. Bobby Kannan, R.K. Singh Raman. In vitro degradation and mechanical integrity of calcium-containing magnesium alloys in modified-simulated body fluid. Biomaterials, 2008, 29: 2306-14

[5] K.Y. Chiu a, M.H. Wong a, F.T. Cheng, et al. Characterization and corrosion studies of fluoride conversion coating on degradable Mg implants [J]. Surface \& Coatings Technology, 2007, 202: 590-8

[6] Huang Jingjing, Ren Yibin, Zhang Bingchun. Preparation and property of coating on degradable Mg implant [J].The Chinese Journal of Nonferrous Metals,2007,17 (9): 1465-9

[7] Gao Jiacheng, Li Longchuan, Wang yong. Surface modification on magnesium by alkali-heat-treatment and its corrosion behaviors in SBF[J]. The Chinese Journal of Nonferrous Metals,2004,14 (9): 1508-13

[8] Gong Pei, Wang Xinyu, Guo Jie. Preparation of Hydroxyapatite Coating on Magnesium via Biomimetic Synthesis [J].Chemical Intermediate,2008,3: 17-20

[9] Xu Yue, Li Sha. Corrosion Resistance and Mechanism of Cerium Oxide Ceramic Coatings on 
Magnesium Alloys [J].Rare Metal Materials and Engineering,2007,36 (Supplement 2): 725-7

[10] Zeng Rongchang, Song Zhifu, Chen Jun, etc. Magnesium alloy AM60 surface modification and its corrosion in Hank's solution [J]. Journal of Chongqing Institute of Technology,2007,21 (10): 5-9

[11] P. Shi, W.F. Ng, M.H. Wong, et al. Improvement of corrosion resistance of pure magnesium in Hanks' solution by microarc oxidation with sol-gel TiO2 sealing [J]. Journal of Alloys and Compounds, 2008, In press

[12] Y.W. Song , D.Y. Shan, E.H. Han. Electrodeposition of hydroxyapatite coating on AZ91D magnesium alloy for biomaterial application [J]. Materials Letters, 2008, In press

[13] H. Wang, Y. Estrin, Z. ZúberováBio-corrosion of a magnesium alloy with different processing histories [J]. Materials Letters. 2008, 62: 2476-9

[14] Tan Xiaowei, Gao Jiacheng, Wang yong, etc. Heat treatment experimental study on medical pure magnesium [J].Chinese and Technology Review,2006,23 (2): 67-69

[15] Chenglong Liu,Yunchang Xin, Guoyi Tang, et al. Influence of heat treatment on degradation behavior of bio-degradable die-cast AZ63 magnesium alloy in simulated body fluid [J]. Materials Science and Engineering A, 2007, 456: 350-7

[16] Huang Fuqiang, Yu Zhentao, Wei Beiling, etc.The Deformability of Magnesium Alloy AZ31

Rolling at Room Temperature [J]. Rare Metal Materials and Engineering,2008,237-42 